\title{
Magnetic Field Induced Ordering in Quasi-One-Dimensional Quantum Magnets
}

\author{
Stefan Wessel and Stephan Haas \\ Department of Physics and Astronomy, University of Southern California, Los Angeles, CA \\ 90089-0484
}

(April 24, 2018)

\begin{abstract}
Three-dimensional magnetic ordering transitions are studied theoretically in strongly anisotropic quantum magnets. An external magnetic field can drive quasi-one-dimensional subsystems with a spin gap into a gapless regime, thus inducing long-range three-dimensional magnetic ordering due to weak residual magnetic coupling between the subsystems. Compounds with higher spin degrees of freedom, such as N-leg spin-1/2 ladders, are shown to have cascades of ordering transitions. At high magnetic fields, zero-point fluctuations within the quasi-1D subsystems are suppressed, causing quantum corrections to the ordering temperature to be reduced.
\end{abstract}

Compounds with strongly anisotropic crystal structures typically exhibit low-dimensional behavior at high temperatures. At low temperatures, the specific nature of their quantum fluctuations determines whether three-dimensional (3D) ordering |1] or other instabilities [2] occur, or whether there is no transition at all [3]. In particular, weakly coupled Heisenberg spin-1/2 chains are known to have a 3D magnetic ordering transition [1] or a spin-Peierls (SP) instability at low temperatures [2], if there is sufficiently strong coupling with low-lying phonon modes. On the other hand, compounds with an intrinsic spin gap, like e.g. weakly coupled integer-spin chains [的 or even-leg spin-1/2 Heisenberg ladders in a spin-liquid state, retain their one-dimensionality down to zero temperature [5]. The pure RVB nature of their 
groundstate renders them inert to weak residual magnetic couplings between the quasi-1D subsystems. Thus 3D magnetic ordering and SP transitions are suppressed.

In this second class of materials, an applied magnetic field, $h$, can decrease the singlettriplet excitation gap of the quasi-1D subsystem, and eventually drive it into a gapless regime if the field exceeds a critical strength, $h_{c 1}$. A transition to a low-temperature ordered phase due to residual magnetic couplings becomes again possible in this partially polarized regime [6:7]. In this paper, we propose that 3D ordering as a result of the deconfinement of pairs of bound spinons by an external magnetic field can actually be realized in a wide variety of quasi-1D physical systems, including anisotropic spin chains, ladders, and SP compounds. Furthermore, a unified phenomenology for the magnetic phase diagram of these materials is presented, starting from an analysis of weakly coupled antiferromagnetic Heisenberg spin-1/2 chains (AFHC) with an easy-axis anisotropy. Interestingly, we observe that for compounds such as N-leg spin ladders with plateaus in their magnetization curves, $m(h)$, [8] a cascade of $\mathrm{N} / 2$ ordering transitions for $\mathrm{N}$ even and $(\mathrm{N}+1) / 2$ transitions for $\mathrm{N}$ odd occurs at high magnetic fields.

We are especially interested in the application of this magnetic field induced ordering transition to the material $\mathrm{Cu}_{2}\left(\mathrm{C}_{2} \mathrm{H}_{12} \mathrm{~N}_{2}\right)_{2} \mathrm{Cl}_{4}(\mathrm{CuHpCl})$ [9]. Typically, the spin gap in most of the ladder compounds known to date is too large to be overcome by presently available magnetic fields. However, this particular material has only a small spin gap of $\approx 10.5$ Kelvin which makes the interesting gapless regime experimentally accessible. It has recently been pointed out that $\mathrm{CuHpCl}$ may better be modeled by an ensemble of weakly coupled dimers than as an antiferromagnetic 2-leg ladder [10]. Whatever the precise magnetic structure may turn out to be, a magnetic field induced ordering transition can occur in all quasi-1D spin systems with a singlet-triplet excitation gap, including Ising-like chains, spin-Peierls chains, and ensembles of spin dimers. Other possible candidate materials with (relatively small) spin gaps include $\mathrm{KCuCl}_{3}$ [11], $\mathrm{CuGeO}_{3}$ [2], $\alpha^{\prime}-\mathrm{NaV}_{2} \mathrm{O}_{5}$ [12], and the homologous series of cuprates $\mathrm{Sr}_{n-1} \mathrm{Cu}_{n+1} \mathrm{O}_{2 n}$ [5].

Let us first consider a crystal of weakly coupled anisotropic antiferromagnetic spin-1/2 


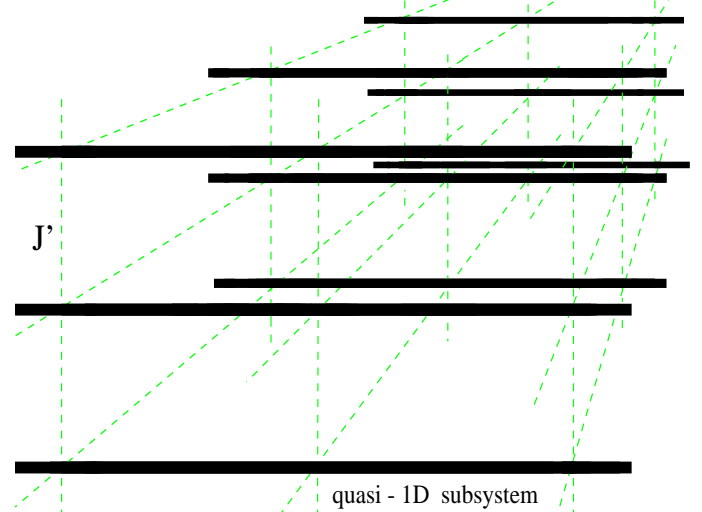

FIG. 1. Schematic illustration of a strongly anisotropic 3D crystal, containing quasi-1D subsystems, e.g. antiferromagnetic spin ladders. The coupling $J^{\prime}$ between the $1 \mathrm{D}$ elements is small compared to the exchange constant $J$ within the subsystems. Therefore the compound has quasi-1D properties at high temperatures $\left(T \gg J^{\prime}\right)$, while at low temperatures a $1 \mathrm{D}$ to $3 \mathrm{D}$ ordering transition can occur if the 1D subsystems are (driven) gapless.

Heisenberg chains, described by the Hamiltonian

$$
H^{1 D}=\sum_{i}\left[J\left(S_{i}^{x} S_{i+1}^{x}+S_{i}^{y} S_{i+1}^{y}+\Delta S_{i}^{z} S_{i+1}^{z}\right)-h S_{i}^{z}\right]
$$

where $J>0$ is the antiferromagnetic exchange constant within the chains, $\Delta$ is an easy-axis anisotropy, and $h$ is an applied external magnetic field. The chains are weakly coupled by $H^{\prime}=J^{\prime} \sum_{<i, j>} \mathbf{S}_{i} \cdot \mathbf{S}_{j}$ with $0<J^{\prime} \ll J$. At zero magnetic field, the excitation spectrum of $H^{1 D}$ is gapless in the XY regime $(\Delta<1)$ and massive in the Ising regime $(\Delta>1)$, with a Kosterlitz-Thouless transition at the Heisenberg point $(\Delta=1)$ [13. In the Ising regime, a finite critical magnetic field, $h_{g}$, has to be overcome to completely soften the lowest triplet mode at wavevector $\pi$, and to drive the system gapless.

From a numerical solution of the Bethe Ansatz integral equations of Eq. 1 [14], we have obtained the magnetic field dependent spinon velocity, $u(h)$, the Luttinger exponent, $K(h)$, the magnetization, $m(h)$, and the radius of compactification, $R(h)$, for the effective low-energy $\mathrm{c}=1$ conformal field theory. Magnetization curves, $m(h)$, for an XY-like and an Ising-like anisotropic Heisenberg chain are shown in Fig. 2. In the dispersion curves of the 


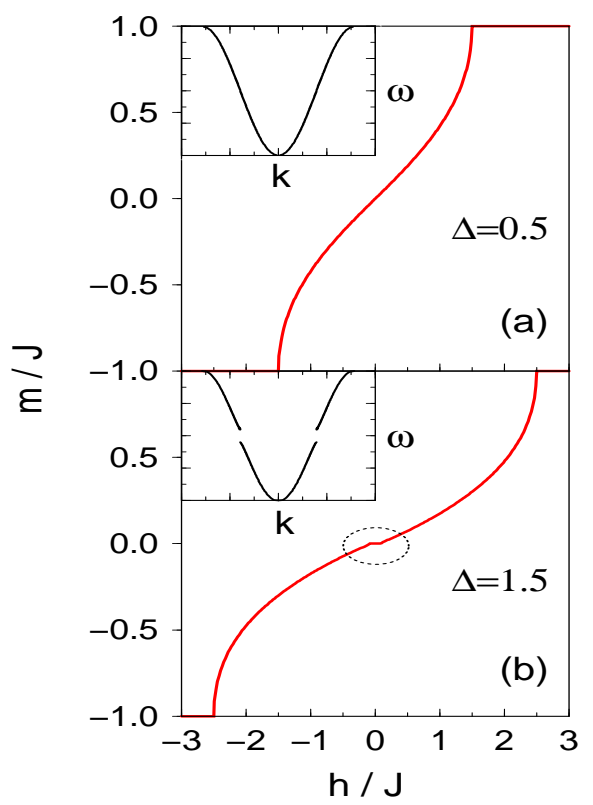

FIG. 2. Magnetization curve, $m(h)$, of an antiferromagnetic spin- $1 / 2$ XXZ chain. (a) XY regime with Ising anisotropy: $\Delta=0.5$, and (b) Ising regime with $\Delta=1.5$. The insets show the dispersion law, $\omega(k)$, for the corresponding continuum models. Here the magnetic field acts as a chemical potential, rising from the bottom of the band at $h_{c 1}$ to the top at $h_{c 2}$. In the Ising regime, a spin gap opens around $\mathrm{h}=0$, leading to a plateau in $m(h)$.

equivalent spinless-fermion model (insets of Fig. 2), the external magnetic field corresponds to a chemical potential. Below $h_{c 1}=-J(1+\Delta)$ the cosine band, $\omega(k)$, of the spinless fermions is empty, and $u\left(h_{c 1}\right)=(\partial \omega / \partial k)_{h_{c 1}}=0$. At the special field $h=0$, the quasilong-range ordered spin density wave within the chains becomes commensurate with the lattice, and Umklapp processes open up a spin gap for $\Delta>1$, leading to a plateau in the magnetization curve (Fig. $2(\mathrm{~b}))$ for $-h_{g}<h<h_{g}$, with

$$
h_{g}=2 \pi J \frac{\sinh \gamma}{\gamma} \sum_{n=0}^{\infty}\left[\cosh \left(\frac{(2 n+1) \pi^{2}}{2 \gamma}\right)\right]^{-1},
$$

where $\Delta=\cosh (\gamma)$ [15]. Beyond $h_{c 2}=J(1+\Delta)$, the chains are fully polarized in the direction of the applied magnetic field. The particle-hole symmetry about $h=0$ is reflected by the shape of $m(h)$ and the 3D ordering temperature, $T_{c}(h)$ (Fig. 3), discussed below.

The low-temperature behavior of the susceptibility in the gapless regime is determined by the dominant low-frequency spinon modes at momentum $q_{z}=\pi$ : 


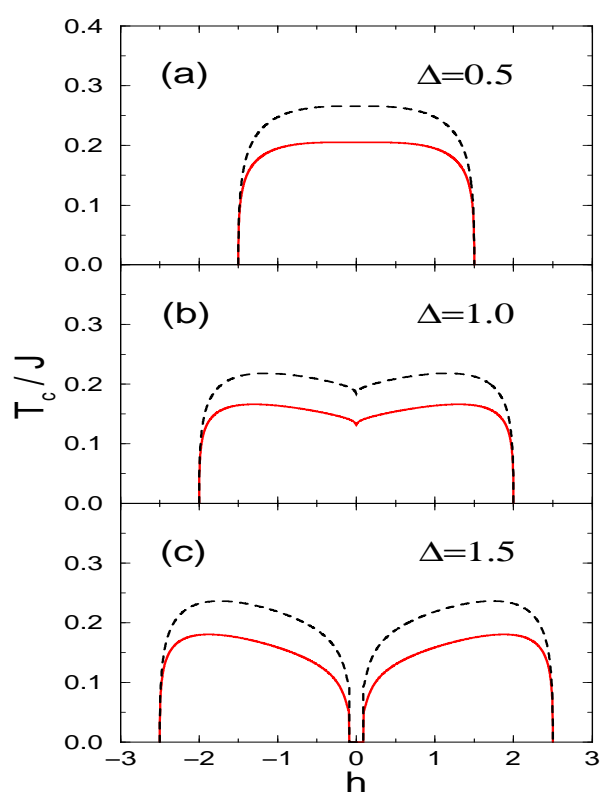

FIG. 3. 3D ordering temperature (solid line) as a function of the applied magnetic field in a cubic crystal of weakly coupled antiferromagnetic spin- $1 / 2$ chains. (a) XY regime with $\Delta=0.5$, (b) Heisenberg point $(\Delta=1.0)$, and Ising regime with $\Delta=1.5$. The dashed lines indicate the onset of the fluctuation region below which the $3 \mathrm{D}$ magnetic correlation length becomes comparable to the inter-chain spacing. For this plot, we have chosen $J^{\prime} / J=1 / 16$.

$$
\begin{aligned}
\chi_{+-}^{1 D}\left(q_{z}, \omega\right. & =0 ; T)=F(\Delta)\left[\frac{\sin \left(\frac{\pi}{4 K}\right)}{u}\left(\frac{2 \pi T}{u}\right)^{\frac{1}{2 K}-2}\right. \\
& \left.\times B^{2}\left(\frac{1}{8 K}, 1-\frac{1}{4 K}\right)-\frac{\pi}{u(1-1 / 4 K)}\right],
\end{aligned}
$$

where $B(x, y)$ is Euler's beta function, and $F(\Delta)$ is a prefactor which strongly depends on the Ising anisotropy [16]. Here, it has been assumed that the chains are parallel to the $z$-axis of the crystal. Furthermore, we have neglected higher-order logarithmic corrections which arise in a more rigorous treatment of the backscattering processes.

Within the mean field approximation of Ref. [17], the low-temperature divergence in $\chi^{1 D}\left(q_{z}, \omega=0 ; T\right)$ drives a 3D ordering transition due to the weak inter-chain couplings, $J^{\prime}$ :

$$
\chi^{3 D}(\mathbf{q}, \omega=0 ; T)=\frac{\chi^{1 D}\left(q_{z}, \omega=0 ; T\right)}{1+J^{\prime} f(\mathbf{q}) \chi^{1 D}\left(q_{z}, \omega=0 ; T\right)},
$$

where $f(\mathbf{q})$ is the crystal form factor which we here set to $f(\mathbf{q})=-1$ for simplicity (simple cubic lattice). The transition temperature, $T_{c}$, is determined from the locus of divergence 
of $\chi^{3 D}(\mathbf{q}, \omega=0 ; T)$. In Fig. 3, the magnetic field dependence of $T_{c}$ is shown in the critical regime $(\Delta=0.5)$, at the marginal point $(\Delta=1)$, and in the massive region $(\Delta=1.5)$. As the easy-axis anisotropy - equivalent to a nearest-neighbor repulsion in the spinless fermion picture - is increased, Umklapp scattering processes lead to a suppression of $T_{c}(h)$ around $h=0$. In the Ising regime these processes become relevant, and a spin gap opens up, causing $T_{c}$ to vanish for $|h|<h_{g}$.

To scrutinize the quality of this approach, we have calculated the Gaussian fluctuations which decrease the value of the transition temperature, $T_{c}(h)$, obtained from the mean field calculation [18]. This suppression is found to be rather small $(\mathcal{O}(1 \%))$ because the corresponding Ginzburg-Landau parameter is proportional to the ratio $J^{\prime} / J \ll 1$. We thus conclude that the mean field treatment gives a quantitatively adequate description of the ordering transition. Furthermore, the onset of the fluctuation region below which the 3D magnetic correlation length becomes comparable to the inter-chain lattice spacing is indicated by the dashed line in Fig. 3. This precursor region is quite large $(\mathcal{O}(\approx 20 \%))$, indicating a sizable staggered magnetization, $m_{s}$, in the ordered regime. Clearly, zero-point fluctuations are exponentially suppressed by $J^{\prime}$.

Let us now examine magnetic field induced 3D ordering transitions in quasi-1D systems with additional internal degrees of freedom, such as weakly coupled Heisenberg spin-S chains with $\mathrm{S}>1 / 2, \mathrm{~N}-\operatorname{leg} \mathrm{S}=1 / 2$ ladders with $\mathrm{N} \geq 2$, and spin-Peierls compounds.

The low-energy Hamiltonian for a SP chain in a magnetic field is given by

$$
H^{S P}=\sum_{i}\left[J\left(1+\delta(-1)^{i}\right) \mathbf{S}_{i} \cdot \mathbf{S}_{i+1}-h S_{i}^{z}\right]
$$

where $\delta$ is the effective lattice distortion due to the coupling between spinon and phonon degrees of freedom. There are three well-known regimes in this system: (i) for $|h|<h_{c 1}$, it is in a spin-liquid phase with a spin gap $\Delta_{S G} \simeq \delta^{2 / 3}|\log \delta|^{-1 / 2}$ [19 for sufficiently small $\delta$ 's, and $h_{c 1}=\mathcal{O}\left(\Delta_{S G}\right)$; (ii) for $h_{c 1}<|h|<h_{c 2}$ the system is a gapless spin-density wave with a field-dependent modulation; (iii) for $|h|>h_{c 2}$ it is fully polarized in the direction of the applied magnetic field. 
In the gapless, partially polarized, region (ii), we can map the low-energy spectrum of this system onto an effective XY-like Heisenberg chain (Eq. 1) with the parameters: $J_{e f f}=J(1-\delta) / 2, \Delta_{e f f}=1 / 2$, and $h_{e f f}=h-J(5+3 \delta) / 4$. This mapping is valid for $\delta \lesssim 1$. The lower and upper critical fields of the gapless regime are $h_{c 1}=J(1+3 \delta) / 2$ and $h_{c 2}=$ $2 J$ [20]. Considering weak antiferromagnetic 3D couplings between the SP chains, $H^{\prime}=$ $J^{\prime} \sum_{<i, j>} \mathbf{S}_{i} \cdot \mathbf{S}_{j}$, the qualitative magnetic field dependence of the $3 \mathrm{D}$ ordering temperature, $T_{c}(h)$, is thus found to be the same as in Fig. 3(a).

As seen in the above example, weakly coupled anisotropic Heisenberg chains in a field can serve as an effective theory for other quasi-1D quantum magnets with richer internal structure. Let us now extend this idea to weakly coupled N-leg spin-1/2 Heisenberg ladders with the Hamiltonian

$$
H^{N}=J_{\|} \sum_{\leftrightarrow} \mathbf{S}_{i, \tau} \cdot \mathbf{S}_{j, \tau}+J_{\perp} \sum_{\downarrow} \mathbf{S}_{i, \tau} \cdot \mathbf{S}_{i, \tau^{\prime}},-h \sum_{i, \tau} S_{i, \tau}^{z},
$$

where $\mathrm{i}$ and $\mathrm{j}$ enumerate the rungs, $\tau, \tau^{\prime}$ label the legs, and the sum marked by $\leftrightarrow(\uparrow)$ runs over nearest neighbors along legs (rungs). Ladders with even and odd number of legs are known to have quite distinct features at zero field $(h=0)$ : at low energies, odd-leg systems can be mapped onto effective gapless antiferromagnetic Heisenberg chains with longer-range interactions [21]. In even-leg systems, relevant inter-band scattering processes open up a spin gap for any finite positive inter-chain coupling, $J_{\perp}>0$ [22] This is the reason why approximations based on strong coupling anisotropies, i.e. expansions in $J_{\|} / J_{\perp}$ [8.23], give a correct qualitative picture, extending even beyond the isotropic $\left(J_{\|}=J_{\perp}\right)$ regime [8,22]. Furthermore, many physical ladder materials show coupling anisotropies within the ladder complex, e.g. a recent structural analysis of the candidate vanadate ladder material $\mathrm{NaVa}_{2} \mathrm{O}_{5}$ suggests a strong rung-coupling anisotropy of $J_{\|} / J_{\perp} \approx 13 / 75$ [24], and for the proposed 2-leg cuprate ladder, $\mathrm{CuHpCl}$, the anisotropy ratio was estimated to be $J_{\|} / J_{\perp} \approx 1 / 5$ [25].

It has recently been shown that N-leg Heisenberg spin-1/2 ladders can have up to N/2 plateaus in their magnetization curve, $m(h)$, if $\mathrm{N}$ is even, and $(\mathrm{N}+1) / 2$ plateaus if $\mathrm{N}$ is odd 
TABLE I. Parameters of the effective low-energy model for the gapless regions of N-leg spin-1/2 ladders in a magnetic field, $h$. The effective magnetic field is given by $h_{\text {eff }}=h-h_{c}(0) J_{\perp}-c_{h} J_{\|}$.

\begin{tabular}{|l|l|l|l|l|}
\hline \hline$N$ & $J_{e f f} / J_{\|}$ & $\Delta_{e f f}$ & $h_{c}(0)$ & \multicolumn{1}{l}{$c_{h}$} \\
\hline 2 & 1 & 0.5 & 1 & 0.5 \\
\hline 3 & $(1,1)$ & $(1,0.5)$ & $(0,1.5)$ & $(0,0.5)$ \\
\hline 4 & $(1.075,1)$ & $(0.3489,0.375)$ & $(0.6589,1.7071)$ & $(0.375,0.625)$ \\
\hline 5 & $(1.0169,1.0961,1)$ & $(1,0.3789,0.3)$ & $(0,1.1189,1.809)$ & $(0,0.2958,0.7)$ \\
\hline 6 & $(1.1114,1.1348,1)$ & $(0.3163,0.3011,0.25)$ & $(0.4916,1.386,1.866)$ & $(0.3515,0.3985,0.75)$ \\
\hline 7 & $(1.0344,1.1415$, & $(1,0.3407$, & $(0,0.8848$, & $(0,0.2166$, \\
& $1.1663,1)$ & $0.2381,0.2143)$ & $1.5504,1.901)$ & $0.4891,0.7857)$ \\
\hline 8 & $(1.1364,1.1882$ & $(0.302,0.2743$, & $(0.3926,1.1506$, & $(0.3432,0.2888$, \\
& $1.1917,1)$ & $0.1962,0.1875)$ & $1.6577,1.9239)$ & $0.5555,0.8125)$ \\
\hline \hline
\end{tabular}

8]. These plateaus are related to gaps between the spin multiplets in the excitation spectra. The partially polarized regions between the plateaus are gapless, and can be described by effective massless $\mathrm{c}=1$ conformal field theories, corresponding to a spin- $1 / 2$ AFHC Hamiltonian with parameters $\left(J_{e f f}, \Delta_{e f f}, h_{e f f}\right)$. In table 1 , the numerical values of these parameter sets, obtained from a strong rung expansion $\left(J_{\|} / J_{\perp} \ll 1\right)$ are listed for $\mathrm{N}=2, \ldots, 8$ legs.

Applying the mean field approach described above to a crystal of weakly coupled N-leg ladders, we now observe a cascade of $\mathrm{N} / 2((\mathrm{~N}+1) / 2) 3 \mathrm{D}$ ordering transitions for quasi-1D ladder subsystems with an even (odd) number of legs, shown in Fig. 4. In the case of weakly coupled even-leg ladders, the first transition is driven by the formation of a quasi-long-range ordered SDW of triplets along the ladder direction, commensurate with the magnetic field, $h>h_{c 1}$. The following transition (for $N>2$ ) is driven by a SDW of quintuplets, etc. . Depending on the ratio $J_{\|} / J_{\perp}$, these phases of different multiplet polarization may overlap, and mixed phases can occur (see e.g. Fig. $4(\mathrm{~d})$ around $h=1.5 J_{\perp}$ and 1.8 $J_{\perp}$ ). The resulting 3D ordering temperature does not vanish completely in this case, but has minima 


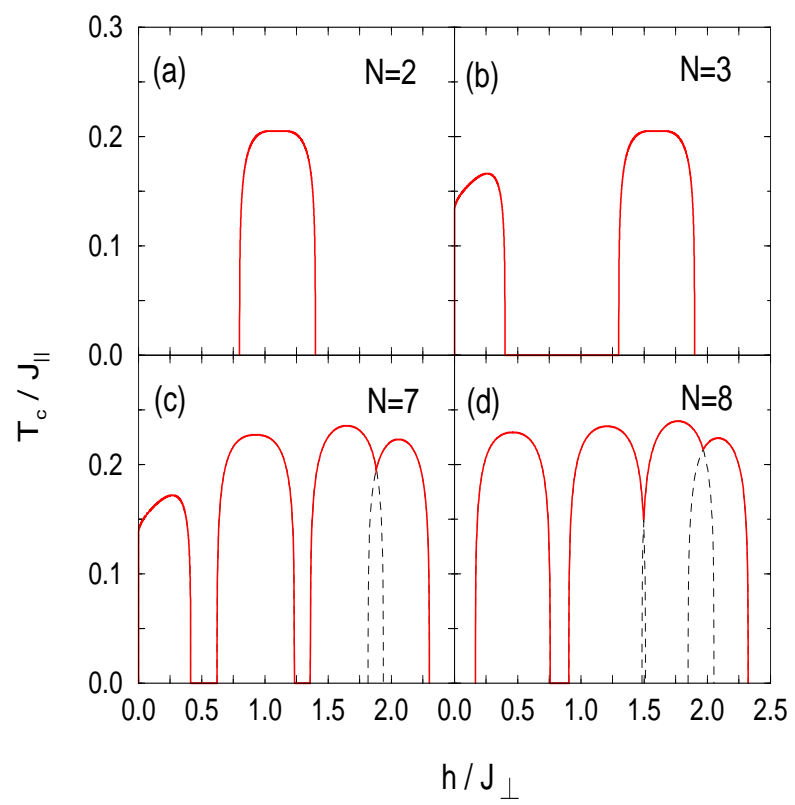

FIG. 4. 3D ordering transition temperatures of N-leg spin-1/2 Heisenberg ladders as a function of an external magnetic field. Cascades of transitions are observed for $N>2$, driven by 1D SDW's of spin-S multiplets on the ladders. For this plot, we have chosen an anisotropy ratio $J_{\|} / J_{\perp}=1 / 5$ and residual inter-ladder couplings $J^{\prime} / J_{\|}=1 / 16$.

at particular magnetic fields where the number of the lower multiplet excitations equals the number of the next-higher multiplet excitations. By analogy, a cascade in $T_{c}(h)$ should occur in compounds containing weakly coupled integer-spin Heisenberg spin chains.

Odd-leg spin-1/2 ladders - as well as half-odd-integer-spin Heisenberg chains - also have a sequence of ordering transitions, with the only difference that the onset of the first transition occurs already at $h=0$ (Figs. $4(\mathrm{~b})$ and $(\mathrm{c})$ ). The dip features in $T_{c}(h)$, due to strong Umklapp scattering $\left(\Delta_{e f f} \lesssim 1\right)$ become less pronounced at larger magnetic fields, i.e. $\Delta_{\text {eff }} \rightarrow$ 0 . This is expected because the effective spin degrees of freedom of the higher multiplets are more "classical" as $S \rightarrow \infty$, and quantum fluctuations are suppressed at high fields. Finally, as the width of the ladder subsystems is increased, the gaps between the various multiplets disappear, and a quasi-continuous $T_{c}(h)$-curve emerges - as it is expected for the limiting case $(N=\infty)$ of weakly coupled 2 D Heisenberg planes.

In summary, we have examined 3D magnetic ordering transitions in quasi-1D materials. 
In the presence of a spin gap, an external magnetic field can drive the 1D subsystems gapless, thus inducing long-range 3D magnetic ordering due to residual magnetic coupling between the subsystems. For compounds with higher spin degrees of freedom, such as N-leg spin-1/2 ladders, cascades of ordering transitions are predicted to occur, reflecting the mesoscopic character of these materials. At high magnetic fields, quantum fluctuations in the quasi-1D subsystems are suppressed, causing the "dip feature" in $T_{c}(h)$ - due to Umklapp processes to disappear for the transitions at higher fields.

We wish to thank G. Bickers, C. Broholm, E. Dagotto, A. Honecker, D. Scalapino, and A. Tsvelik for useful discussions, and acknowledge the Zumberge Foundation for financial support. S.W. is partially supported by DAAD grant No. HSP III,D/98/11174. 


\section{REFERENCES}

[1] D.A. Tennant et al., Phys. Rev. Lett. 70, 4003 (1993); Phys. Rev. B 52, 13368 (1995).

[2] M. Hase, I. Terasaki, and K. Uchiokura, Phys. Rev. Lett. 70, 3651 (1993).

[3] Y. Imry, P. Pincus, and D. Scalapino, Phys. Rev. B 12, 1978 (1975).

[4] S. Ma et al., Phys. Rev. Lett. 69, 3571 (1992).

[5] M. Azuma et al., Phys. Rev. Lett. 73, 3463 (1994); Z. Hiroi, M. Azuma, M. Takano, and Y. Baudo, J. Solid State Chem. 95, 230 (1991).

[6] S. Haas and M. Sigrist, to be published in Physical Phenomena at High Magnetic Fields III, edited by J.R. Schrieffer, L. Gorkov, and Z. Fisk, World Scientific, Singapore (1999).

[7] T. Giamarchi and A. M. Tsvelik, Phys. Rev. B 59, 11398 (1999).

[8] D. C. Cabra, A. Honecker, and P. Pujol, Phys. Rev. Lett. 79, 5126 (1997); Phys. Rev. B 58, 6241 (1998).

[9] G. Chaboussant et al., Phys. Rev. Lett. 80, 2713 (1998); Eur. Phys. J. B 6, 167 (1998).

[10] C. Broholm, private communications.

[11] N. Cavadini et al., Eur. Phys. J. B 7, 519 (1999).

[12] D. Augier et al., Phys. Rev B 56, R5732 (1997).

[13] A. Luther, Phys. Rev B 14, 2153 (1976).

[14] N.M. Bogoliubov, A.G. Izergin, and V.E. Korepin, Nucl. Phys. B 275, 687 (1986).

[15] J. des Cloizeaux and M. Gaudin, J. Math. Phys. 7, 1384 (1966).

[16] H.J. Schulz, Phys. Rev. B 346372 (1986); S.Lukyanov and A.B. Zamolokchiov, Nucl. Phys. B 493, 571 (1997).

[17] H.J. Schulz, Phys. Rev. Lett. 77, 2790 (1996). 
[18] N. Menyhard, J. Phys. C 11, 2207 (1978); H.J. Schulz and C. Bourbonnais, Phys. Rev. B 27, 5856 (1983); R. H. McKenzie, Phys. Rev. B 51, 6249 (1995)

[19] A. Klümper, Euro. Phys. J. B 5, 677 (1998).

[20] In the case of an additional next nearest neighbor interaction, $J_{2}$, the parameters are $J_{e f f}=J(1-\delta)-J_{2}, \Delta_{e f f}=\left(J(1-\delta)+J_{2}\right) / 2\left(J(1-\delta)-J_{2}\right), h_{e f f}=h-J(5+3 \delta) / 4-J_{2} / 2$.

[21] B. Frischmuth, S. Haas, G. Sierra, and T. M. Rice, Phys. Rev. B 55, R3340 (1997).

[22] S.P. Strong and A.J. Millis, Phys. Rev. Lett. 69, 2419 (1992).

[23] F. Mila, Eur. Phys. J. B 6, 210 (1998); K. Tandon et al., Phys. Rev. B 59396 (1999).

[24] P. Horsch and F. Mack, Eur. Phys. J. B 5, 367 (1998).

[25] C.A. Hayward, D. Poilblanc, and L.P. Levy, Phys. Rev. B 54, R12649 (1996). 